\title{
Relação entre Instabilidade Hidrodinâmica e velocidade de queda por Simulação Numérica Direta
}

\author{
Celso Menoti da Silvalt \\ UFRGS, Porto Alegre, RS \\ Edith Beatriz Camaño Schettini] \\ UFRGS, Porto Alegre, RS
}

\begin{abstract}
Resumo. Em estuários é comum o surgimento de correntes de turbidez que podem ser classificadas como hipopicnais, no qual a densidade da água do rio carregada de sedimentos é menor que a água do mar. Nesse contexto surgem fenômenos de instabilidade hidrodinâmica na interface entre as correntes. O presente trabalho investigou a relação entre a topologia da instabilidade hidrodinâmica na interface das concentrações com a velocidade de queda da partícula de sedimentos, por Simulação Numérica Direta. Por essa metodologia, foi possível estabelecer que a velocidade de queda interfere nos Fluxos Turbulentos de tal forma que possibilita condições para o surgimento de instabilidades com topologia semelhante à da Instabilidade de Rayleigh-Taylor.
\end{abstract}

Palavras-chave. Simulação Numérica, Intabilidade Hidrodinâmica, Velocidade de Queda, Transporte de Sedimentos

\section{Introdução}

Os rios são responsáveis por despejar milhões de toneladas de sedimentos diariamente nos oceanos. A água doce, que entra no oceano, carregada de partículas, é tipicamente menos densa que a água salgada circundante. Assim, à medida que os rios entram nos oceanos, uma série de fenômenos são responsáveis pelo transporte de sedimentos. O deslocamento e a dispersão de manchas de poluentes ou nuvens de partículas são relevantes em estudos de impacto ambiental. Tais fenômenos de transporte podem ser ocasionados pela turbulência e/ou por diferença de densidade ou temperatura entre o fluido ambiente e a corrente transportada, conhecidos como correntes de densidade. Segundo Lowe 7 (1982), uma corrente de densidade em um líquido ou gás se mantém em movimento devido a ação da força de gravidade, que atua sobre a diferença de densidade. Nesse contexto, o efeito do empuxo pode gerar instabilidades hidrodinâmicas que poderão interferir no transporte e sedimentação. Uma instabilidade característica dessa configuração é a instabilidade de Rayleigh-Taylor, que depende da diferente de densidade entre os campos. Os trabalhos de Burns e Meiburg apresentaram resultados relevantes sobre a instabilidade em corrente de densidade. Dentre os resultados, destacam-se a Análise Instabilidade Linear [1] e a Simulação Numérica Direta da corrente de densidade [2]. Outra configuração de grande relevância é a Camada de Mistura, caracterizada pelo perfil médio de velocidade horizontal com um ponto de inflexão. Dessa conformação surge necessariamente a instabilidade hidrodinâmica de Kelvin-Helmholtz.

A presente investigação simulou uma Camada de Mistura Duplamente Estratificada com duas grandezas escalares, concentração de sedimentos e concentração salina, configuração semelhante

\footnotetext{
${ }^{1}$ celso.menoti@ufrgs.br

2 bcamano@iph.ufrgs.br
} 
ao de uma corrente hipopicnal, com o objetivo de verificar a influência da velocidade de queda na formação de instabilidades e investigar as causas dessa relação. Os diâmetros de partículas considerados no campo de sedimentos correspondem às velocidades de queda adimensionais de $u_{s}=0$ até $u_{s}=0,05[9]$. Foram analisadas os formas topológicas da instabilidade na interface dos campos e relacionado ao comportamento dos Fluxos Turbulentos com a finalidade de caracterizar as formas topológicas das instabilidade de Kelvin-Helmholtz e Rayleight-Taylor, segundo a influencia da velocidade de queda da partícula de sedimentos.

\section{Metodologia}

O presente trabalho considerou uma camada de mistura com dois perfis escalares, em que a concentração de sedimentos é instável e a concentração salina é estável. A massa específica do fluido, $\rho$, é uma função das concentrações escalares, $\varphi_{1}$ e $\varphi_{2}$, de sedimentos e de salinidade, respectivamente, e é expressa por:

$$
\rho=\rho_{0}\left(1+\alpha_{1} \varphi_{1}+\alpha_{2} \varphi_{2}\right),
$$

em que $\rho_{0}$ é a massa específica da água clara, $\alpha_{1}$ e $\alpha_{2}$ são os coeficientes de expansão volumétrica da concentração de sedimentos e da salinidade, respectivamente. A variação de massa específica total foi considerada significativa apenas no termo de empuxo na Equação de Navier-Stokes, conforme a Hipótese de Boussinesq [1].

As grandezas características usadas na adimensionalização foram: $L=\delta_{u}, U=\Delta u / 2, \varphi_{1, \max }$ e $\varphi_{2, \max }$. Como consequência, surgem os números adimensionais de Reynolds, Re, de Richardson, $R i$, e de Schmidt, $S c$, que estão definidos na Tabela 1. As equações adimensionalizadas ficam:

$$
\begin{aligned}
\frac{\partial u_{i}}{\partial x_{i}} & =0 \\
\frac{\partial u_{j}}{\partial t}+u_{i} \frac{\partial u_{j}}{\partial x_{i}} & =-\frac{\partial p}{\partial x_{j}}+\frac{1}{R e} \frac{\partial^{2} u_{j}}{\partial x_{i} \partial x_{i}}+R i_{2}\left(\frac{1}{R_{\rho}} \varphi_{1}+\varphi_{2}\right) \delta_{i 2}, \\
\frac{\partial \varphi_{1}}{\partial t}+\left(u_{i}+u_{s} \delta_{i 2}\right) \frac{\partial \varphi_{1}}{\partial x_{i}} & =\frac{1}{R e S c_{1}} \frac{\partial^{2} \varphi_{1}}{\partial x_{i} \partial x_{i}} \\
\frac{\partial \varphi_{2}}{\partial t}+u_{i} \frac{\partial \varphi_{2}}{\partial x_{i}} & =\frac{1}{R e S c S c_{2}} \frac{\partial^{2} \varphi_{2}}{\partial x_{i} \partial x_{i}}
\end{aligned}
$$

Tabela 1: Grupos adimensionais e definições segundo a adimensionalização proposta. As constantes $\kappa_{1}$ e $\kappa_{2}$ correspondem à difusividade da concentração de partículas e salina, respectivamente.

\begin{tabular}{ll}
\hline Adimensional & Definição \\
\hline Número de Reynolds & $R e=\delta_{u} U / \nu$ \\
Número de Richardson & $R i_{k}=\alpha_{k} \varphi_{k, \max } g \delta_{u} / U^{2}$ \\
Número de Schmidt & $S c_{k}=\nu / \kappa_{k}$ \\
Razão de Densidade & $R_{\rho}=\alpha \varphi_{2, \max } / \beta \varphi_{1, \max }$ \\
\hline
\end{tabular}

O índice $k$ indica a grandeza escalar: $k=1$ corresponde à concentração de partículas e $k=2$ à concentração salina. Nos experimentos numéricos adotou-se $R e=1000, R i_{2}=0,4, R_{\rho}=1$ e $S c_{1}=S c_{2}=1$. A Razão de Densidade $\left(R_{\rho}\right)$ também corresponde à razão dos Números de Richardson salino e da concentração de partículas.

Para simular a camada de mistura temporal foram adotadas as seguintes condições de contorno: 
- Condição Periódica para todas as variáveis na direção do eixo $0 x_{1}$ : se $F$ é uma variável do problema, então $F\left(x_{1}=0, x_{2}, x_{3}, t\right)=F\left(x_{1}=L_{x_{1}}, x_{2}, x_{3}, t\right)$;

- Condição de deslizamento livre: para $x_{2}= \pm L_{x_{2}} / 2$, tem-se $\partial u_{1} / \partial x_{2}=0$ e $u_{2}=0$.

- Para a condição de contorno no limite superior, $x_{2}=L_{x} / 2$ pressupõe-se que o fluxo de massa é nulo. Com efeito, a integração da Equação (4), em regime permanente, resulta em:

$$
u_{s} \varphi_{1}+\frac{1}{S c R e} \frac{\partial \varphi_{1}}{\partial x_{2}}=0
$$

conhecida também como condição de Robin [1. Considerou-se ainda que não há fluxo através da fronteira superior: $\partial \varphi_{2} / \partial x_{2}=0$.

Os Perfis do Fluxo Base, cujo esquema é apresentado na Figura 1, correspondem à condição inicial da simulação em que $h=u_{s} t=0$ :

- Velocidade: $u_{1}\left(x_{1}, x_{2}, x_{3}, 0\right)=\bar{u}_{1}\left(x_{2}\right)$ e $u_{2}\left(x_{1}, x_{2}, x_{3}, 0\right)=u_{3}\left(x_{1}, x_{2}, x_{3}, 0\right)=0$.

- Concentrações: $\varphi_{1}\left(x_{1}, x_{2}, x_{3}, 0\right)=\bar{\varphi}_{1}\left(x_{2}\right)$ e $\varphi_{2}\left(x_{1}, x_{2}, x_{3}, 0\right)=\bar{\varphi}_{2}\left(x_{2}\right)$.
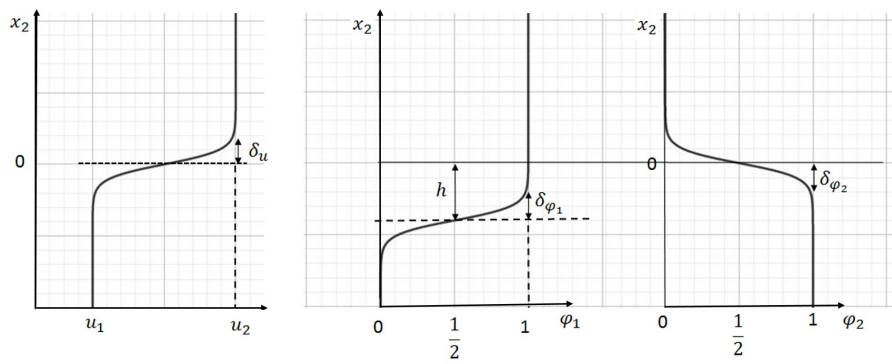

Figura 1: Perfis de base: o gráfico da esquerda corresponde ao perfil de velocidade média; os gráficos da direita apresentam os perfis escalares: concentração de sedimentos $\left(\varphi_{1}\right)$ e a concentração de sedimentos $\left(\varphi_{2}\right)$.

As Equações dos Perfis de Base adotadas são:

$$
\begin{aligned}
& \bar{u}_{1}\left(x_{2}\right)=U \tanh \left(\frac{2 x_{2}}{\delta_{u}}\right), \\
& \bar{\varphi}_{1}\left(x_{2}\right)=\frac{\varphi_{1, \max }}{2}\left[1+\tanh \left(\frac{2 x_{2}}{\delta_{\varphi_{1}}}\right)\right], \\
& \bar{\varphi}_{2}\left(x_{2}\right)=\frac{\varphi_{2, \max }}{2}\left[1-\tanh \left(\frac{2 x_{2}}{\delta_{\varphi_{2}}}\right)\right],
\end{aligned}
$$

sendo, $U=\left(u_{2}+u_{1}\right) / 2, \delta_{u}, \delta_{\varphi_{1}}$ e $\delta_{\varphi_{2}}$ correspondem à metade das espessuras dos perfis da camada de mistura da velocidade, concentração de sedimentos e concentração salina. Com o objetivo de favorecer a instabilidade na interface do escoamento, no instante inicial é adicionado ao perfil de velocidade de base um ruído branco com amplitude $A_{f}$, descrito por funções trigonométricas que satisfazem a Equação da Continuidade (3) 8 [ 9 . Segundo o estudo de análise de estabilidade para camada de mistura, o modo de instabilidade predominante é o modo de Kelvin-Helmholtz [3]. O dimensionamento do domínio de cálculo deve ser uma função do comprimento de onda do modo 
mais amplificado, $\lambda$, na direção principal do escoamento 8] 9]. O domínio de cálculo adotado foi: $0 \leq x_{1} \leq L_{x_{1}}$ e $-L_{x_{1}} / 2 \leq x_{2} \leq L_{x_{1}} / 2$ sendo que $L_{x_{1}}=7$. Para as simulações a malha adotada foi $n_{x_{1}}=256, n_{x_{2}}=521$ e $\Delta t=0,001$.

A metodologia numérica adotada foi a Simulação Numérica Direta (DNS, Direct Numerical Simulation). O código utilizado é o Incompact3d, 4], que permite a resolução numérica das equações de Navier-Stokes, Continuidade e Advecção-Difusão, para escoamentos incompressíveis, utilizando esquemas compactos de sexta ordem para a discretização espacial. A integração no tempo é feita pelo método de Adams-Bashforth de terceira ordem. As equações do movimento são resolvidas em uma malha cartesiana, uniforme em cada direção. Maiores detalhes podem ser encontrados em Laizet et al (2011) 5].

Com o crescimento da amplitude das perturbações, os termos não lineares passam a influenciar na instabilidade com a formação de vórtices característicos à forma topológica. O comportamento dos campos escalares médios, ao longo do tempo, pode ser representado pelas equações:

$$
\begin{aligned}
\frac{\partial \bar{\varphi}_{1}}{\partial t}-u_{s} \frac{\partial \bar{\varphi}_{1}}{\partial x_{2}} & =\frac{1}{R e S c_{1}} \frac{\partial^{2} \bar{\varphi}_{1}}{\partial x_{2}^{2}}-\frac{\partial}{\partial x_{1}}\left(\overline{u_{1}^{\prime} \varphi_{1}^{\prime}}\right)-\frac{\partial}{\partial x_{2}}\left(\overline{u_{2}^{\prime} \varphi_{1}^{\prime}}\right) \\
\frac{\partial \bar{\varphi}_{2}}{\partial t} & =\frac{1}{R e S c_{2}} \frac{\partial^{2} \bar{\varphi}_{2}}{\partial x_{2}^{2}}-\frac{\partial}{\partial x_{1}}\left(\overline{u_{1}^{\prime} \varphi_{2}^{\prime}}\right)-\frac{\partial}{\partial x_{2}}\left(\overline{u_{2}^{\prime} \varphi_{2}^{\prime}}\right) .
\end{aligned}
$$

Inicialmente os termos não lineares são de baixa ordem reduzindo essas equações ao problema de Difusão Unidimensional. Esse período inicial pode ser denominado de período linear e sua descrição é apresentada por Silva [9].

A quantificação da não linearidade pode ser estimada pelos Fluxos Turbulentos. Definiu-se como Fluxo Turbulento do escalar $k$ na direção $j$, à expressão:

$$
F_{k j}=\int_{\Omega} \varphi_{k}^{\prime}\left(\mathbf{u}^{\prime} \cdot \mathbf{e}_{j}\right) d S
$$

Os Fluxos turbulentos são medidas que permite estimar a Difusividade Turbulenta de um escalar $k, \kappa_{t k}$. Segundo Smyth e Kimura (2010) [10, $\kappa_{t k}$ é calculada no domínio por:

$$
\kappa_{t k}=-\frac{\int_{-L_{x_{2}} / 2}^{L_{x_{2} / 2}} F_{k}\left(x_{2}, t\right) d x_{2}}{\int_{\Omega} \frac{\partial \bar{\varphi}_{k}}{\partial x_{2}} d \Omega} .
$$

A razão dos Coeficientes de Difusividade Turbulenta $\left(\tau_{t}=\kappa_{t 1} / \kappa_{t 2}\right)$ foi utilizada para comparar a diferença dessa grandeza nos campos escalares.

\section{Resultados}

Os casos simulados diferenciaram-se apenas pela velocidade de queda, $u_{s}$. Os valores considerados foram: $u_{s}=0,0,001$ e 0,01, 0,025 e 0,050. As situações em que $u_{s} \leq 0,01$ apresentaram comportamento idêntico ao caso sem velocidade de queda, $u_{s}=0$, com predomínio absoluto da instabilidade de Kelvin-Helmoholtz que é formada na interface entre os campos escalares. Nos casos em que $u_{s} \geq 0,025$, o desenvolvimento da instabilidade indica que a topologia da instabilidade de Kelvin-Helmoholtz começa a ser modificada no campo de sedimentos, com surgimento de outra topologia (Figura 2).

Para o caso no qual $u_{s}=0$, os perfis médios escalares (Figura 3 mantiveram o padrão simétrico ímpar, mesmo com o crescimento da não linearidade. Para o instante $t=34,8$, por exemplo, o perfil apresenta a mesma forma do perfil inicial médio e será solução das Equações (10) e (11). Com o crescimento das parcelas não lineares no tempo, ocorre a deformação do perfil médio inicial 
mantendo a simetria. Dessa forma, os fluxos turbulentos verticais são iguais e a difusividade turbulenta nos campos escalares é a mesma.
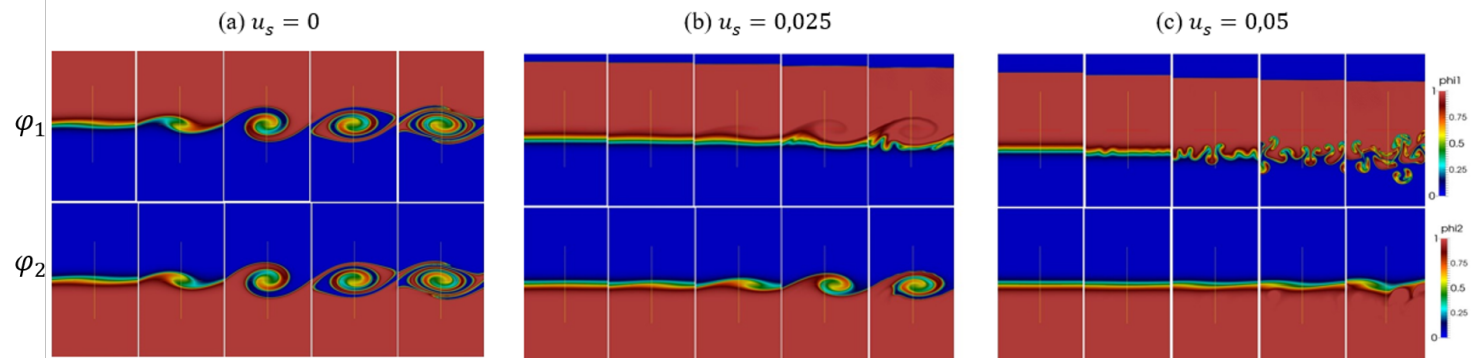

Figura 2: Sequência de desenvolvimento temporal para os instantes $34.8,40.2,45.6,51$, e 56.4 da camada de mistura para $R_{\rho}=1$ nos casos: (a) $u_{s}=0$; (b) $u_{s}=0,025$; (c) $u_{s}=0,050$.
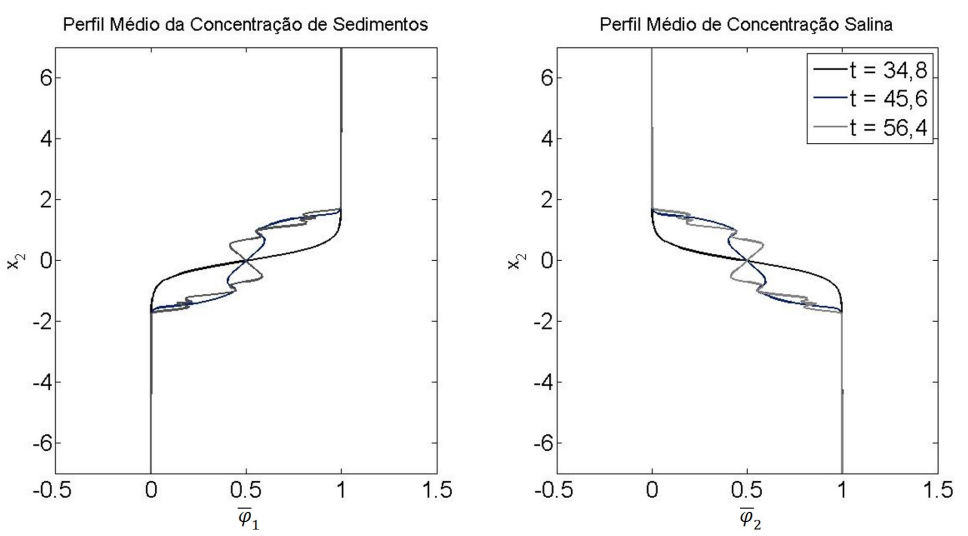

Figura 3: Evolução dos perfis escalares médios para o caso em que $R_{\rho}=1$, sem sedimentação $u_{s}=0$.

Os casos em que a velocidade de queda $u_{s} \geq 0,025$, os perfis escalares médios não apresentam simetria em relação ao eixo vertical, $\overrightarrow{0 x_{2}}$, ao longo do tempo (Figura 4). Tal comportamento justifica-se pela Equação (10), visto que a advecção vertical depende da velocidade de sedimentação. Como consequência, o fluxo vertical turbulento do campo de sedimentação, aumenta a difusividade turbulenta desse campo. Na simulação em que $u_{s}=0,050$, a sequência de campos de concentração de sedimentos (Figura 2) indica o surgimento de uma forma topológica similar à Instabilidade de Rayleigh-Taylor. Pela evolução apresentada pelos casos em que $u_{s}=0,025$ e $u_{s}=0,050$, atribui-se essa forma topológica à velocidade de queda.

Quando $0 \leq u_{s} \leq 0,01$, a topologia instável característica é a de Kelvin-Helmholtz, pois o perfil médio de velocidade horizontal mantém um ponto de inflexão, condição necessária para a formação dessa instabilidade [6]. Portanto, espera-se que o fluxo turbulento horizontal seja predominante. Para avaliar a relação entre os Fluxos Turbulentos, foi proposta a Razão de Fluxos Turbulentos Médios do campo de sedimentos, em termos absolutos $\left(\zeta=F_{1 x_{1}} / F_{1 x_{2}}\right)$, em que $F_{1 x_{p}}$ corresponde à média espacial do fluxo turbulento na direção $p \in 1,2$, na qual 1 e 2 correspondem às direções horizontal e vertical, respectivamente, e os Fluxos Turbulentos são calculados pela Equação (12).

Conforme o previsto, para os casos em que há um predomínio da Instabilidade de KelvinHelmholtz o Fluxo turbulento horizontal é predominante e a Razão de Difusividade Turbulenta é 
(a)

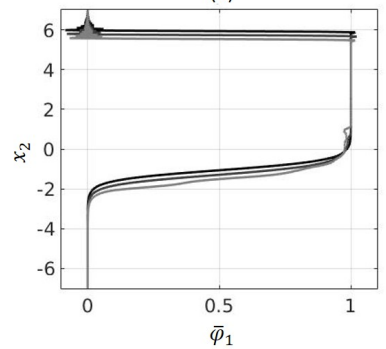

(c)

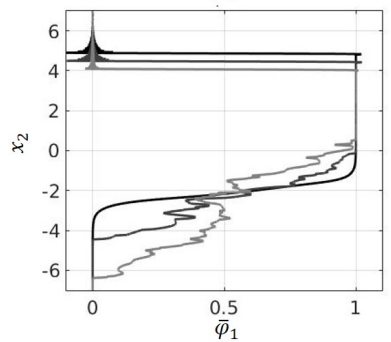

(b)

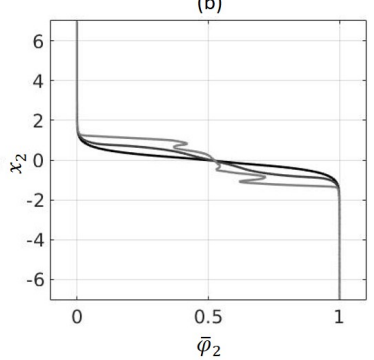

(d)

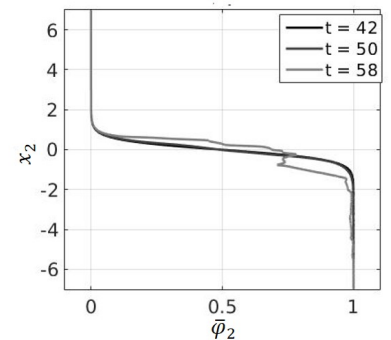

Figura 4: Perfis médio espaciais escalares para os casos em que $R_{\rho}=1,0$ com velocidade de queda de $u_{s}=0,025$, gráficos (a) e (b), e $u_{s}=0,05$, gráficos (c) e (d), para os campos de sedimentos $\left(\bar{\varphi}_{1}\right)$ e salinidade $\left(\bar{\varphi}_{1}\right)$, respectivamente.
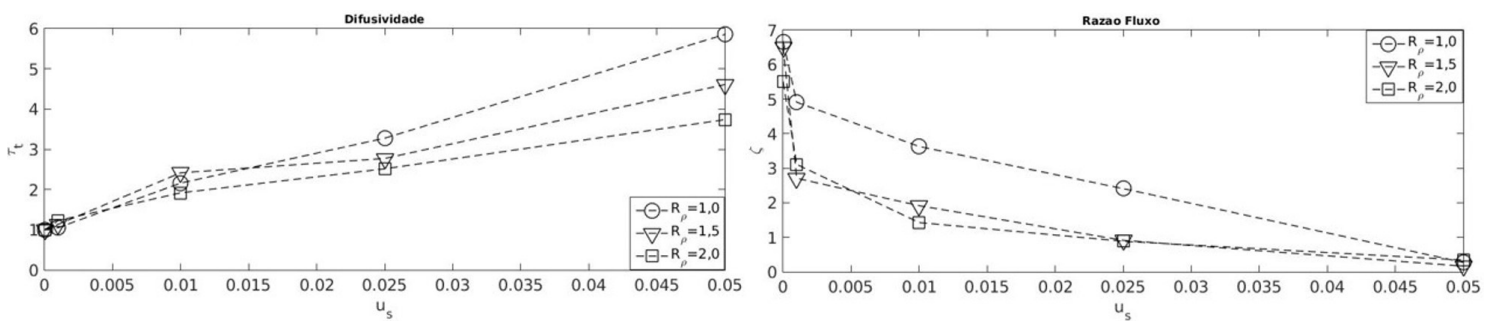

Figura 5: Relações entre Difusividade Turbulenta Média, $\tau_{t}$, e a Razão de Fluxo Médio, $\zeta$, com a velocidade de queda $\left(u_{s}=0,10^{-3}, 10^{-2}, 2,5 \times 10^{-2}\right.$ e $\left.5 \times 10^{-2}\right)$.

próxima da unidade (Figura 5). Já nos casos em que $u_{s}=0,025$ e 0,050, o fluxo turbulento vertical torna-se predominante e a Difusividade Turbulenta do campo de sedimentos cresce, principalmente quando $u_{s}=0,05$, caso em que surge uma topológica similar à Instabilidade de Rayleigh-Taylor na interface.

\section{Conclusões}

O presente trabalho investigou a relação entre a velocidade de queda e a forma topológica da instabilidade por Simulação Numérica Direta. Os casos simulados variaram a velocidade de queda sem variação de densidade, ou seja $R_{\rho}=1$. Os campos escalares gerados indicaram que para velocidade de queda menores $u_{s}=0,01$, há um predomínio da instabilidade de Kelvin-Helmholtz na interface dos campos. Verificou-se que nesses casos a difusividade turbulenta é próxima da unidade e predomina o Fluxo Turbulento Horizontal. Para o caso em que a velocidade de queda 
é $u_{s}=0,050$, a forma topológica verificada na interface é semelhante à Instabilidade de RayleighTaylor. Verificou-se, também, um crescimento dos Fluxos Turbulentos Verticais e da difusividade turbulenta no campo de sedimentos. Portanto, a velocidade de queda da partícula interfere na dinâmica da formação de instabilidades hidrodinâmicas e modifica a forma típica da instabilidade da camada de mistura.

\section{Referências}

[1] Burns, P., Meiburg, E. , Sediment-laden fresh water above salt water: linear stability analysis Journal of Fluid Mechanics, Volume 691, pages 279-314, 2012. DOI: 10.1017/jfm.2011.474

[2] Burns, P., Meiburg, E. , Sediment-laden fresh water above salt water: non-linear simulations Journal of Fluid Mechanics, Volume 762, pages 156-195, 2015. DOI: 10.1017/jfm.2014.645

[3] Farenzena, B. A., Silvestrini, J. H., Linear stability analysis of particle-laden hypopycnal plumes Physics of Fluids, Volume 29, pages 124108-1 - 124108-9, 2017. DOI: 10.1063/1.4999343

[4] INCOMPACT3D. Incompact3d, 2020. Página Inicial. Disponível em $<$ www.incompact3d.com $>$. Acesso em 12 de março ce 2020.

[5] Laizet, S., Li, N., A powerful tool to tackle turbulence problems with up to $O\left(10^{5}\right)$ ) computational cores Int. J. Numer. Meth. Fluids, Volume 67, pages 1735 - 1757, 2011. DOI: $10.1002 /$ fld. 2480

[6] Lesieur, M., Turbulence in Fluids. Kluwer Academic Publishers, Netherlands, 2008.

[7] Lowe, D. R. Sediment gravity flows; II, Depositional models with special reference to the deposits of high-density turbidity currents, Jounal of Sedimentary Petrology, Volume 52, pages 279-297, 1982. DOI: 10.1306/212F7F31-2B24-11D7-8648000102C1865D

[8] Martinez, D. M. V. Transição à Turbulência na Camada de Mistura Estavelmente Estratificada utilizando Simulação Numérica Direta e de Grandes Escalas, Tese de Doutorado, UFRGS, 2006.

[9] Silva, C. M., Simulação numérica de instabilidades hidrodinâmicas em camada de mistura duplamente estratificada com efeito de velocidade de queda, Tese de Doutorado, UFRGS, 2019.

[10] Smyth, W. D., Li, Kimura, S., Mixing in a Moderately Sheared Salt-Fingering Layer J. of Physical Oceanography, Volume 41, pages 1364 - 1384, 2010. DOI: 10.1175/2010JPO4611.1 